Research Article

\title{
A result on the strength of graphs by factorizations of complete graphs
}

\author{
Rikio Ichishima $^{1, *}$, Francesc A. Muntaner-Batle ${ }^{2}$, Akito Oshima $^{2}$ \\ ${ }^{1}$ Department of Sport and Physical Education, Faculty of Physical Education, Kokushikan Universityty, 7-3-1 Nagayama, Tama-shi, Tokyo 206-8515, \\ Japan \\ ${ }^{2}$ Graph Theory and Applications Research Group, School of Electrical Engineering and Computer Science, Faculty of Engineering and Built Environment, \\ The University of Newcastle, NSW 2308, Australia
}

(Received: 21 September 2021. Received in revised form: 14 December 2021. Accepted: 15 December 2021. Published online: 23 December 2021.)

(C) 2021 the authors. This is an open access article under the CC BY (International 4.0) license (www.creativecommons.org/licenses/by/4.0/).

\begin{abstract}
A numbering $f$ of a graph $G$ of order $n$ is a labeling that assigns distinct elements of the set $\{1,2, \ldots, n\}$ to the vertices of $G$. The strength of $G$ is defined by

$$
\operatorname{str}(G)=\min \left\{\operatorname{str}_{f}(G) \mid f \text { is a numbering of } G\right\},
$$

where $\operatorname{str}_{f}(G)=\max \{f(u)+f(v) \mid u v \in E(G)\}$. In this paper, some results obtained from factorizations of complete graphs are presented. In particular, it is shown that for every $k \in[1, n-1]$, there exists a graph $G$ of order $n$ satisfying $\delta(G)=k$ and $\operatorname{str}(G)=n+k$, where $\delta(G)$ denotes the minimum degree of $G$.
\end{abstract}

Keywords: strength; factorization; graph labeling; combinatorial optimization.

2020 Mathematics Subject Classification: 05C70, 05C78, 90C27.

\section{Introduction}

In this paper, only finite graphs without loops or multiple edges are considered. Terms and notation not defined below follow those used in [2].

The vertex set of a graph $G$ is denoted by $V(G)$, while the edge set of $G$ is denoted by $E(G)$. The path and the complete graph of order $n$ are denoted by $P_{n}$ and $K_{n}$, respectively. The degree of a vertex $v$ in a graph $G$ is the number of edges of $G$ incident with $v$, which is denoted by $\operatorname{deg} v$. The minimum degree of $G$ is the minimum degree among the vertices of $G$ and is denoted by $\delta(G)$. A graph $G$ is $r$-regular if $\operatorname{deg} v=r$ for each $v \in V(G)$.

The bandwidth problem was originated in the 1950s in the form of finding a matrix equivalent to a given matrix so that the non-zero entries lie within a narrow band about the main diagonal. Afterwards, Harper [10] investigated the bandwidth numberings of hypercubes; these related to the error-correcting codes subject to minimizing the maximum absolute error. Since then a considerable amount of papers have been published on this subject (see surveys [3,4,15]).

For the sake of brevity, we denote the interval of integers $k$ such that $i \leq k \leq j$ by simply writing $[i, j]$. A numbering $f$ of a graph $G$ of order $n$ is a labeling that assigns distinct elements of the set $[1, n]$ to the vertices of $G$. The bandwidth of $G$ is defined by

$$
\text { band }(G)=\min \left\{\operatorname{band}_{f}(G) \mid f \text { is a numbering of } G\right\} \text {, }
$$

where $\operatorname{band}_{f}(G)=\max \{|f(u)-f(v)| \mid u v \in E(G)\}$. An additive analogous for bandwidth numberings of graphs has been introduced in [11] as a generalization of the problem of finding whether a graph is super edge-magic or not (see [6] for the definition of a super edge-magic graph, and also consult either [1] or [7] for alternative and often more useful definitions of the same concept). The strength of $G$ is defined by

$$
\operatorname{str}(G)=\min \left\{\operatorname{str}_{f}(G) \mid f \text { is a numbering of } G\right\},
$$

where $\operatorname{str}_{f}(G)=\max \{f(u)+f(v) \mid u v \in E(G)\}$.

Several sharp bounds for the strength of a graph have been found in terms of other parameters defined on graphs (see [11] and [13]). Among others, the following result established in [11] that provides a lower bound for the strength of a graph in terms of its order and minimum degree is particularly useful. 
Lemma 1.1. For every graph $G$ of order $n$ with $\delta(G) \geq 1$,

$$
\operatorname{str}(G) \geq n+\delta(G) .
$$

For further knowledge on the strength of graphs, the authors suggest that the reader consult the results in [9, 12,14]. There are other related parameters that have been studied in the area of graph labelings. Excellent sources for more information on this topic are found in the extensive survey by Gallian [8], which also includes information on other kinds of graph labeling problems as well as their applications.

\section{Connections with factorizations of complete graphs}

The $k$ th power $G^{k}$ of a graph $G$, where $k \geq 1$, is the graph which has the same vertex set as $G$ and in which $u v$ is an edge if and only if $u$ and $v$ are connected in $G$ by a path of length at most $k$. Chvátal [5] proved that a graph $G$ of order $n$ has bandwidth $k(k \in[1, n-1])$ if and only if $k$ is the smallest positive integer for which $G$ is a subgraph of $P_{n}^{k}$. In other words, it can be stated as follows.

Theorem 2.1. For every $k \in[1, n-1]$, there exists a graph $G$ of order $n$ satisfying band $(G)=k$.

In this section, we establish strength analogues to the bandwidth result given in Theorem 2.1.

At this point, it is convenient to introduce some additional concepts and notation. A graph $G$ is said to be factorable into the factors $F_{1}, F_{2}, \ldots, F_{t}$ if these factors are pairwise edge-disjoint and $\bigcup_{i=1}^{t} E\left(F_{i}\right)=E(G)$. If $G$ is factored into $F_{1}, F_{2}, \ldots, F_{t}$, then we represent this by $G=F_{1} \oplus F_{2} \oplus \cdots \oplus F_{t}$, which is called a factorization of $G$. A $k$-regular factor of a graph $G$ is a $k$-factor of $G$. If there exists a factorization of a graph $G$ such that each factor is a $k$-factor (for a fixed $k$ ), then $G$ is $k$-factorable.

The following result is considered as a part of mathematical folklore (see [2, p.273] for instance).

Theorem 2.2. For every positive integer $n$, the complete graph $K_{2 n}$ is 1-factorable.

The following proof is inspired by the factorization of $K_{2 n}$ that Chartrand and Lesniak [2] carried out when describing Theorem 2.2.

Theorem 2.3. For every $k \in[2 n+1,4 n-1]$, there exists a graph $G$ of order $2 n$ satisfying $\delta(G) \geq 1$ and $\operatorname{str}(G)=k$.

Proof. There is only one numbering $f$ of $K_{2 n}$ and the label of the edge joining the vertices labeled $2 n$ and $2 n-1$ is $4 n-1$. Thus, $\operatorname{str}_{f}\left(K_{2 n}\right)=\operatorname{str}\left(K_{2 n}\right)=4 n-1$. For that reason, it suffices to construct a graph $G$ of order $2 n$ satisfying $\delta(G) \geq 1$ and $\operatorname{str}(G)=k$ when $k \in[2 n+1,4 n-2]$. Since the result is obvious for $n=1$, we may assume that $n \geq 2$. Let $V\left(K_{2 n}\right)=$ $\left\{v_{i} \mid i \in[0,2 n-1]\right\}$ and arrange the vertices $v_{1}, v_{2}, \ldots, v_{2 n-1}$ in a regular $(2 n-1)$-gon, placing $v_{0}$ in the center. Join every two vertices by a straight line segment, thereby producing $K_{2 n}$. For $i \in[1,2 n-1]$, define the 1-factor $F_{i}$ to consist of the edge $v_{0} v_{i}$ together with all those edges perpendicular to $v_{0} v_{i}$. Thus, $E\left(F_{i}\right)=\left\{v_{0} v_{i}\right\} \cup\left\{v_{i-j} v_{i+j} \mid j \in[1, n-1]\right\}$, where each of the subscripts $i-j$ and $i+j$ is expressed as one of the integers $1,2, \ldots,(2 n-1)$ modulo $(2 n-1)$. Then $K_{2 n}=F_{1} \oplus F_{2} \oplus \cdots \oplus F_{2 n-1}$.

With the preceding construction in hand, we now consider the labeling

$$
f: V\left(K_{2 n}\right) \rightarrow[1,2 n]
$$

such that $f\left(v_{i}\right)=1+i(i \in[0,2 n-1])$. Notice that if we let

$$
M_{i}=\max \left\{f(u)+f(v) \mid u v \in E\left(F_{i}\right)\right\},
$$

where $i \in[1,2 n-1]$, then

$$
M_{i}=\max \left\{\left\{f\left(v_{i-j}\right)+f\left(v_{i+j}\right) \mid j \in[1, n-1]\right\} \cup\left\{f\left(v_{0}\right)+f\left(v_{i}\right)\right\}\right\}
$$

so that

$$
M_{i}= \begin{cases}2 n+1+2 i & \text { if } i \in[1, n-1], \\ 2+2 i & \text { if } i \in[n, 2 n-2], \\ 2 n+1 & \text { if } i=2 n-1 .\end{cases}
$$

This implies that $\left\{M_{i} \mid i \in[1,2 n-1]\right\}=[2 n+1,4 n-1]$ is a set of $(2 n-1)$ consecutive integers. Sort the integers $M_{1}, M_{2}$, $\ldots, M_{2 n-1}$ in descending order so that we get $M_{1}^{\prime}, M_{2}^{\prime}, \ldots, M_{2 n-1}^{\prime}$, and let $F_{i}^{\prime}(i \in[1,2 n-1])$ be the 1-factor having the maximum edge label $M_{i}^{\prime}$. Further, let $G_{r}(r \in[1,2 n-2])$ be the graph with

$$
V\left(G_{r}\right)=\left\{v_{i} \mid i \in[0,2 n-1]\right\} \text { and } E\left(G_{r}\right)=E\left(K_{2 n}\right)-\bigcup_{i=1}^{r} E\left(F_{i}^{\prime}\right)
$$


Then $G_{r}(r \in[1,2 n-2])$ is a $(2 n-1-r)$-regular graph of order $2 n$. It follows from Lemma 1.1 that

$$
\operatorname{str}\left(G_{r}\right) \geq 2 n+(2 n-1-r)=4 n-1-r
$$

for $r \in[1,2 n-2]$. On the other hand, $f$ has the property that

$$
\operatorname{str}_{f}\left(G_{r}\right)=\max \left\{f(u)+f(v) \mid u v \in E\left(G_{r}\right)\right\}=M_{r}^{\prime}=4 n-1-r
$$

for $r \in[1,2 n-2]$. This implies that $\operatorname{str}\left(G_{r}\right) \leq 4 n-1-r$ for $r \in[1,2 n-2]$. It remains to observe that $\delta\left(G_{r}\right) \geq 1$ for each $r \in[1,2 n-2]$. Therefore, we conclude that $\operatorname{str}\left(G_{k}\right)=k$ for every $k \in[2 n+1,4 n-2]$.

The construction described in the proof of the preceding theorem is illustrated with Table 1 for $K_{6}$.

Table 1: A 1-factorization of $K_{6}$.

\begin{tabular}{lll}
\hline$F_{i}$ & $E\left(F_{i}\right)$ & $M_{i}$ \\
\hline$F_{1}$ & $\left\{v_{0} v_{1}\right\} \cup\left\{v_{5} v_{2}, v_{4} v_{3}\right\}$ & $M_{1}=f\left(v_{6-i}\right)+f\left(v_{1+i}\right)=9(i \in[1,2])$ \\
$F_{2}$ & $\left\{v_{0} v_{2}\right\} \cup\left\{v_{1} v_{3}, v_{5} v_{4}\right\}$ & $M_{2}=f\left(v_{5}\right)+f\left(v_{4}\right)=11$ \\
$F_{3}$ & $\left\{v_{0} v_{3}\right\} \cup\left\{v_{2} v_{4}, v_{1} v_{5}\right\}$ & $M_{3}=f\left(v_{3-i}\right)+f\left(v_{3+i}\right)=8(i \in[1,2])$ \\
$F_{4}$ & $\left\{v_{0} v_{4}\right\} \cup\left\{v_{3} v_{5}, v_{2} v_{1}\right\}$ & $M_{4}=f\left(v_{3}\right)+f\left(v_{5}\right)=10$ \\
$F_{5}$ & $\left\{v_{0} v_{5}\right\} \cup\left\{v_{4} v_{1}, v_{3} v_{2}\right\}$ & $M_{5}=f\left(v_{0}\right)+f\left(v_{5}\right)=f\left(v_{5-i}\right)+f\left(v_{i}\right)=7(i \in[1,2])$ \\
\hline
\end{tabular}

A graph $G$ is defined to be Hamiltonian if it has a cycle containing all the vertices of $G$. Such a cycle is called a Hamiltonian cycle. A Hamiltonian factorization of a graph $G$ is a factorization of $G$ such that every factor is a Hamiltonian cycle of $G$. The next result concerns factorizations of $K_{2 n+1}$ into Hamiltonian cycles.

Theorem 2.4. For every positive integer $n$, the complete graph $K_{2 n+1}$ is Hamiltonian factorable.

The following proof is inspired by the Hamiltonian factorization of $K_{2 n+1}$ that Chartrand and Lesniak [2] carried out when describing Theorem 2.4 (see [2, p.275]).

Theorem 2.5. For every $k \in[2 n+2,4 n+1]$, there exists a graph $G$ of order $2 n+1$ satisfying $\delta(G) \geq 1$ and $\operatorname{str}(G)=k$.

Proof. There is only one numbering $f$ of $K_{2 n+1}$ and the label of the edge joining the vertices labeled $2 n+1$ and $2 n$ is $4 n+1$. Thus, $\operatorname{str}_{f}\left(K_{2 n+1}\right)=\operatorname{str}\left(K_{2 n+1}\right)=4 n+1$. In light of this, it suffices to construct a graph $G$ of order $2 n+1$ satisfying $\delta(G) \geq 1$ and $\operatorname{str}(G)=k$ for $k \in[2 n+2,4 n]$. Since the result is clear for $n=1$, we may assume that $n \geq 2$. Let $V\left(K_{2 n+1}\right)=\left\{v_{i} \mid i \in[0,2 n]\right\}$ and arrange the vertices $v_{1}, v_{2}, \ldots, v_{2 n}$ in a rectangular $2 n$-gon, placing $v_{0}$ in the center. Join every two vertices by a straight line segment, thereby producing $K_{2 n+1}$. For $i \in[1, n]$, define the edge set of the factor $F_{i}$ to consist of $v_{0} v_{i}, v_{0} v_{n+i}$, all edges parallel to $v_{i} v_{i+1}$ and all edges parallel to $v_{i-1} v_{i+1}$, where each of the subscripts is taken as the integers $1,2, \ldots, 2 n$ modulo $2 n$. Then $K_{2 n+1}=F_{1} \oplus F_{2} \oplus \cdots \oplus F_{n}$, where $F_{i}$ is the Hamiltonian cycle

$$
v_{0}, v_{i}, v_{i+1}, v_{i-1}, v_{i+2}, v_{i-2}, \ldots, v_{n+i-1}, v_{n+i+1}, v_{n+i}, v_{0} .
$$

With the preceding construction in hand, we now consider the labeling

$$
f: V\left(K_{2 n+1}\right) \rightarrow[1,2 n+1]
$$

such that $f\left(v_{i}\right)=1+i(i \in[0,2 n])$. Let $M_{i}=\max \left\{f(u)+f(v) \mid u v \in E\left(F_{i}\right)\right\}$, where $i \in[1, n]$, and sort the integers $M_{1}, M_{2}, \ldots, M_{n}$ in descending order so that we get $M_{1}^{\prime}, M_{2}^{\prime}, \ldots, M_{n}^{\prime}$. Then

$$
M_{i}^{\prime}=\{4 n+1,4 n-1, \ldots, 2 n+3\}
$$

is an arithmetic progression with $n$ terms and common difference -2 . Further, let $F_{i}^{\prime}$ be the Hamiltonian cycle having the maximum edge label $M_{i}^{\prime}$ for $i \in[1, n]$. Since

$$
\left\{f(u)+f(v) \mid u v \in E\left(K_{2 n+1}\right)\right\}=[3,4 n+1]
$$

and

$$
M_{i}^{\prime}=\max \left\{f(u)+f(v) \mid u v \in E\left(F_{i}^{\prime}\right)\right\}=4 n+3-2 i
$$

for $i \in[1, n]$, it follows that $F_{i}^{\prime}$ has the edge labeled $4 n+3-2 i(i \in[1, n])$. With this knowledge in hand, we consider the next two cases. 
Case 1. We will show the result for odd $k \in[2 n+3,4 n-1]$. Let $G_{r}(r \in[1, n-1])$ be the graph with

$$
V\left(G_{r}\right)=\left\{v_{i} \mid i \in[0,2 n]\right\} \text { and } E\left(G_{r}\right)=E\left(K_{2 n+1}\right)-\bigcup_{i=1}^{r} E\left(F_{i}^{\prime}\right)
$$

It follows from Lemma 1.1 that

$$
\operatorname{str}\left(G_{r}\right) \geq(2 n+1)+2(n-r)=4 n+1-2 r
$$

for $r \in[1, n-1]$. On the other hand, $f$ has the property that

$$
\operatorname{str}_{f}\left(G_{r}\right)=\max \left\{f(u)+f(v) \mid u v \in E\left(G_{r}\right)\right\}=M_{r+1}^{\prime}=4 n+1-2 r
$$

for $r \in[1, n-1]$. This implies that $\operatorname{str}\left(G_{r}\right) \leq 4 n+1-2 r$ for $r \in[1, n-1]$. It remains to observe that $\delta\left(G_{r}\right) \geq 1$ for each $r \in[1, n-1]$. Therefore, we conclude that $\operatorname{str}\left(G_{r}\right)=k$ for odd $k \in[2 n+3,4 n-1]$.

Case 2. We will show the result for even $k \in[2 n+2,4 n]$. For $k=4 n$, let $H_{1}$ be the graph with $V\left(H_{1}\right)=\left\{v_{i} \mid i \in[0,2 n]\right\}$ and $E\left(H_{1}\right)=E\left(K_{2 n+1}\right)-\left\{v_{2 n-1} v_{2 n}\right\}$. Then $\delta\left(H_{1}\right)=2 n-1$, which follows by Lemma 1.1 that

$$
\operatorname{str}\left(H_{1}\right) \geq(2 n+1)+(2 n-1)=4 n .
$$

Since $f$ has the property that

$$
\operatorname{str}_{f}\left(H_{1}\right)=\max \left\{f(u)+f(v) \mid u v \in E\left(H_{1}\right)\right\}=M_{1}^{\prime}-1=(4 n+1)-1=4 n,
$$

it follows that $\operatorname{str}\left(H_{1}\right) \leq 4 n$. Thus, $\operatorname{str}\left(H_{1}\right)=4 n$. For even $k \in[2 n+2,4 n-2]$, let $H_{s}(s \in[2, n-1])$ be the graph with

$$
V\left(H_{s}\right)=\left\{v_{i} \mid i \in[0,2 n]\right\} \text { and } E\left(H_{s}\right)=E\left(G_{s-1}\right)-\bigcup_{i=1}^{s}\left\{v_{2 n+1-i} v_{2 n-2 s+i}\right\},
$$

where $G_{s}$ is the $2(n-s)$-regular graph of order $2 n+1$ described in Case 1 . Then $H_{s}(s \in[2, n-1])$ is a graph of order $2 n+1$ with $\delta\left(H_{s}\right)=2 n+1-2 s$. It follows from Lemma 1.1 that

$$
\operatorname{str}\left(H_{s}\right) \geq(2 n+1)+(2 n+1-2 s)=4 n+2-2 s
$$

for $s \in[2, n-1]$. Moreover, $f$ has the property that

$$
\begin{aligned}
\operatorname{str}_{f}\left(H_{s}\right) & =\max \left\{f(u)+f(v) \mid u v \in E\left(H_{s}\right)\right\} \\
& =M_{s}^{\prime}-1=(4 n+3-2 s)-1=4 n+2-2 s
\end{aligned}
$$

for $s \in[2, n-1]$. This implies that $\operatorname{str}\left(H_{s}\right) \leq 4 n+2-2 s$ for $s \in[2, n-1]$. It remains to observe that $\delta\left(H_{s}\right) \geq 1$ for each $s \in[2, n-1]$. Therefore, we conclude that $\operatorname{str}\left(H_{k}\right)=k$ for even $k \in[2 n+2,4 n]$.

The preceding theorem and its proof are illustrated with Table 2 for $K_{7}$.

Table 2: A Hamiltonian factorization of $K_{7}$.

\begin{tabular}{ll}
\hline$F_{i}$ & $M_{i}$ \\
\hline$F_{1}: v_{0}, v_{1}, v_{2}, v_{6}, v_{3}, v_{5}, v_{4}, v_{0}$ & $M_{1}=f\left(v_{7-i}\right)+f\left(v_{2+i}\right)=11(i \in[1,2])$ \\
$F_{2}: v_{0}, v_{2}, v_{3}, v_{1}, v_{4}, v_{6}, v_{5}, v_{0}$ & $M_{2}=f\left(v_{6}\right)+f\left(v_{5}\right)=13$ \\
$F_{3}: v_{0}, v_{3}, v_{4}, v_{2}, v_{5}, v_{1}, v_{6}, v_{0}$ & $M_{3}=f\left(v_{4-i}\right)+f\left(v_{3+i}\right)=9(i \in[1,3])$ \\
\hline
\end{tabular}

It is now possible to obtain the following result from Theorems 2.3 and 2.5.

Theorem 2.6. For every $k \in[n+1,2 n-1]$, there exists a graph $G$ of order $n$ satisfying $\delta(G) \geq 1$ and $\operatorname{str}(G)=k$.

The proofs of Theorems 2.3 and 2.5 supply another result.

Theorem 2.7. For every $k \in[1, n-1]$, there exists a graph $G$ of order $n$ satisfying $\delta(G)=k$ and $\operatorname{str}(G)=n+k$.

\section{Conclusion}

In Theorem 2.7, we have provided strength analogues to the bandwidth result given by Chvátal [5] (see Theorem 2.1) as applications with factorizations of complete graphs. 


\section{Acknowledgments}

The authors are gratefully indebted to Yukio Takahashi for his technical assistance. The authors are also grateful to the anonymous referees of this paper for reviewing it carefully.

\section{References}

[1] B. D. Acharya, S. M. Hegde, Strongly indexable graphs, Discrete Math. 93 (1991) 123-129.

[2] G. Chartrand, L. Lesniak, Graphs \& Digraphs, 3rd Edition, CRC Press, Boca Raton, 1996.

[3] P. Z. Chinn, J. Chvátalová, A. K. Dewdney, N. E. Gibbs, The bandwidth problem for graphs and matrices - a survey, J. Graph Theory 6 (1982) 223-254.

[4] F. R. K. Chung, Labelings of graphs, In: L. W. Beineke, R. J. Wilson (Eds.), Selected Topics in Graph Theory, Vol. 3, Academic Press, New York, 1988, pp. 151-168.

[5] V. Chvátal, A remark on a problem of Harary, Czechoslovak Math. J. 20 (1970) 109-111.

[6] H. Enomoto, A. Lladó, T. Nakamigawa, G. Ringel, Super edge-magic graphs, SUT J. Math. 34 (1998) 105-109.

[7] R. M. Figueroa-Centeno, R. Ichishima, F. A. Muntaner-Batle, The place of super edge-magic labelings among other classes of labelings, Discrete Math. 231 (2001) 153-168.

[8] J. A. Gallian, A dynamic survey of graph labeling, Electron. J. Combin. 24 (2021) \#DS6.

[9] Z. B. Gao, G. C. Lau, W. C. Shiu, Graphs with minimal strength, Symmetry 13 (2021) \#513.

[10] L. H. Harper, Optimal numberings and isoperimetric problems on graphs, J. Combin. Theory 1 (1966) 385-393.

[11] R. Ichishima, F. A. Muntaner-Batle, A. Oshima, Bounds for the strength of graphs, Australas. J. Combin. 72 (2018) $492-508$.

[12] R. Ichishima, F. A. Muntaner-Batle, A. Oshima, The strength of some trees, AKCE Int. J. Graphs Comb. 17 (2020) $486-494$.

[13] R. Ichishima, F. A. Muntaner-Batle, A. Oshima, Y. Takahashi, The strength of graphs and related invariants, Memoirs Kokushikan Univ. Inf. Sci. $41(2020) 1-8$

[14] R. Ichishima, F. A. Muntaner-Batle, A. Oshima, Minimum degree conditions for the strength and bandwidth of graphs, Preprint.

[15] Y. L. Lai, K. Williams, A survey of solved problems and applications on bandwidth, edgesum, and profile of graphs, J. Graph Theory 31 (1999) $75-94$. 